\title{
ERRORS IN HIGH PRECISION SOURCE POSITION DETERMINA-
} TION

\author{
PEDRO ELOSEGUI, JUAN-MARIA MARCAIDE \\ Instituto de Astrofisica de Andalucía, Apdo. 2144, E-18080 Granada, Spain
}

IRWIN I. SHAPIRO

Harvard-Smithsonian Center for Astrophysics, 60 Garden Street, Cambridge, MA 02138, U.S.A.

\begin{abstract}
We have made a detailed analysis of the systematic errors in the determination, from two sets of VLBI observations, of the position of the quasar $1038+528$ A relative to the quasar $1038+528 \mathrm{~B}$. This analysis confirms an apparent proper motion at $\lambda=3.6 \mathrm{~cm}$ of $26 \pm 8 \mu \mathrm{as} / \mathrm{yr}$ of the core of the quasar $1038+528 \mathrm{~A}$ relative to the quasar $1038+528 \mathrm{~B}$.
\end{abstract}

\section{INTRODUCTION}

The relative separation of the pair of quasars $1038+528$ A and B is only 33" and, since they can be observed simultaneously at every telescope at $\lambda=3.6 \mathrm{~cm}$, the differenced-phase observable for these sources is almost free of systematic effects introduced by the propagation medium and the instrumentation, thus allowing their relative separation to be determined with microarcsecond precision. The ionospheric error contribution should be no more than one microaresecond (Bartel et al. 1986, Morabito et al. 1986). The tropospheric contribution, more difficult to estimate, should be still smaller. We present a thorough analysis of the systematic errors associated with the estimate of the position of the core of the source $1038+528$ A relative to the source $1038+528 \mathrm{~B}$.

\section{OBSERVATIONS AND DATA ANALYSIS}

We observed this pair of quasars on 17-18 March 1981 and 10-11 May 1983, at $\lambda=3.6$ and $13 \mathrm{~cm}$ wavelengths simultaneously. Details of these observations and of the hybrid mapping of the sources were provided by Marcaide et al. (1985) and Marcaide, Elósegui, and Shapiro (1990).

For these two epochs, we determined for $\lambda=3.6 \mathrm{~cm}$ the positions of the peaks of brightness on the maps of $1038+528 \mathrm{~A}$ (which coincide with the positions of the core) and estimated the separations of these peaks of brightness from a reference point on the $1038+528$ B maps, chosen to be the same point for both epochs. The estimates are given in Table I. The error bar for each estimate corresponds to the statistical standard error, scaled to make $\chi^{2}$ per degree of 
TABLE I Estimated position offsets of the core of $1038+528$ A with respect to the reference position on $1038+528 \mathrm{~B}$.

\begin{tabular}{lrr}
\hline Epoch & $\Delta \alpha \cos \delta(\mu a s)$ & $\Delta \delta(\mu a s)$ \\
\hline 1981.2 & $0.0 \pm 3.0$ & $0.0 \pm 4.1$ \\
1983.4 & $-19.4 \pm 3.3$ & $53.5 \pm 4.0$ \\
\hline
\end{tabular}

freedom unity. Figure $1 a$ shows the two estimates. Thus, we inferred an apparent proper motion at $\lambda=3.6 \mathrm{~cm}$ of $26 \pm 3 \mu \mathrm{ss} / \mathrm{yr}$ of the core of the quasar $1038+528 \mathrm{~A}$ relative to the quasar $1038+528 \mathrm{~B}$.

\section{SYSTEMATIC ERRORS}

An obvious source of systematic error in the source-position determination is the error in the determination of the source reference-point. Every source map can be viewed as a superposition of the true source map and an error map. This error map arises from errors in the calibration of the visibilities, from inadequate $u v$ plane sampling, and from signal-to-noise ratio limits. We have tried to estimate the magnitude of the systematic errors in the source-position determination introduced by errors in the calibration of the visibilities by: (a) assuming that our best source map is the true source map, (b) introducing error maps of various magnitudes, and (c) for each resulting source map, estimating the position of the peak of brightness of the core with respect to a chosen reference point in the quasar $1038+528 \mathrm{~B}$.

To investigate the effect of random calibration errors we used the following procedure: We

(1) made our "best" map of $1038+528$ A after a number of hybrid-mapping iterations. The first step in all iterations was a self-calibration;

(2) took the final fringe amplitudes and phases with antenna-based corrections, and introduced amplitude and phase errors by multiplying each amplitude datum by a random number selected from the range, $1-X$ to $1+X$, and by adding to each phase datum a random phase selected separately from the range, $-Y$ to $+Y$ (in radians);

(3) Fourier inverted and CLEANed the resulting "visibility data";

(4) defined the peak brightness for astrometric purposes as the centroid position of all the $\delta$-CLEAN components whose flux density is greater than $25 \%$ of the flux density of the largest $\delta$-CLEAN component; and

(5) from a weighted-least-squares adjustment to the observed interferometricphase-differences, estimated the angular distance between the peak of brightness of the quasar $1038+528 \mathrm{~A}$ and the feature chosen on the quasar $1038+528 \mathrm{~B}$ as the reference.

We repeated this procedure for several values of $X$ and $Y$, ranging from 0.15 to 0.45 .

To investigate the effect of the uv-plane sampling, we used reduced, disjoint VLBI data sets, and followed a procedure similar to the one described above. 

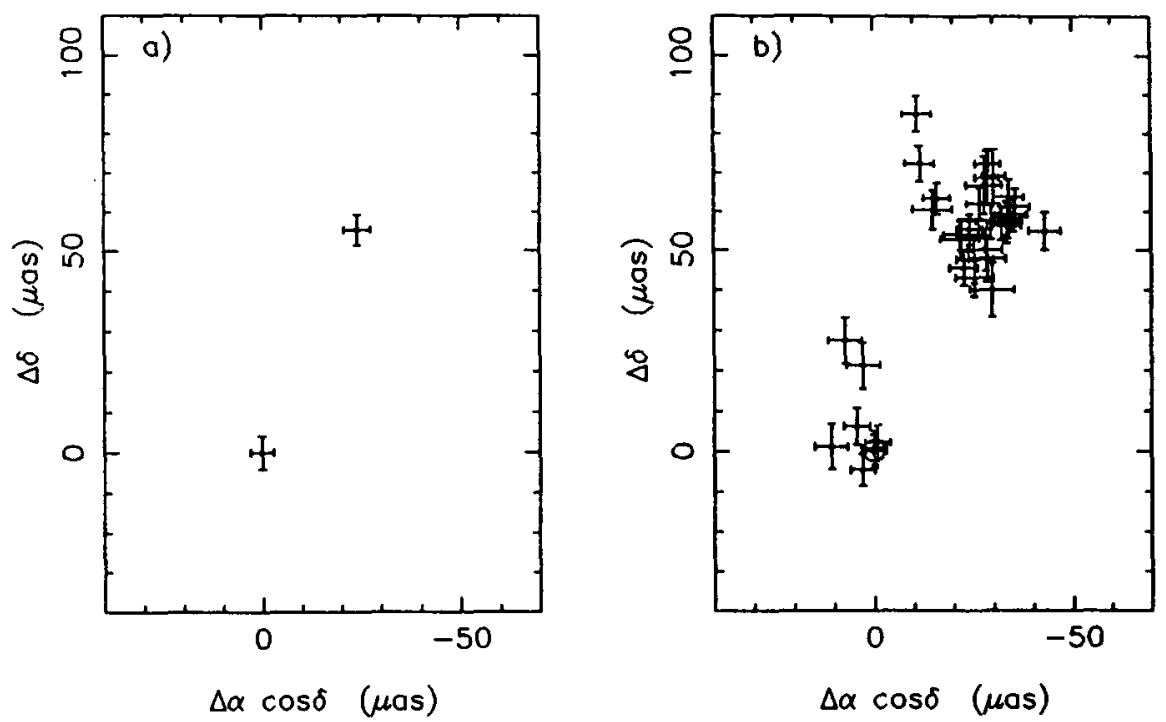

Figure 1. Estimates of the offsets of the position at $\lambda=3.6 \mathrm{~cm}$ of the core of quasar $1038+528 \mathrm{~A}$, relative to $1038+528 \mathrm{~B}$ at epochs 1981.2 and 1983.4 (with 1981.2 offsets substracted): (1a) our best estimate, corresponding to Table I; (1b) summary of all results from the numerical experiments described in the text.

We formed four subsets of the VLBI visibility data of $1038+528 \mathrm{~A}$ and made a map from each, as in step 1, by using data obtained before an arbitrary UT value (placed near the middle of the experiment); the data obtained after that UT value; data for every UT but from only some of the antennas; and data obtained from every UT from the remaining antennas. We then skipped step 2 and proceeded through steps 3 and 4 . In step 5, we used: (5a) the complete set of VLBI phase-difference data with each of the four maps; (5b) the four separate sets of VLBI phase-difference data with our best map of $1038+528 \mathrm{~A}$; and (5c) the four sets of VLBI phase-difference data with their corresponding maps.

We made a further attempt to estimate the effect on the astrometric solution due to systematic errors in our maps. We followed steps 1 , (skipped step $2), 3$, and 4, and in step 5, for each epoch, we removed the structure contributions of quasars $1038+528 \mathrm{~A}$ and $\mathrm{B}$ using our best maps of both epochs. If we label the phase-difference data from epoch 1981.2 and 1983.4 as D1 and D2 and the maps for $1038+528$ A and B from these epochs as A1 and A2 and B1 and B2, respectively, then, in step 5 we estimated the position of the peak of brightness of the core of quasar $1038+528 \mathrm{~A}$ with respect to the chosen reference-point in $1038+528 \mathrm{~B}$ for the following combinations: D1A1B2, D1A2B1, D1A2B2, D2A1B1, D2A1B2, D2A2B1. Figure $1 b$ includes these results, which are not at all sensitive to our choice of $25 \%$ in step 4 of the procedure described above. 
Another possible source of systematic error in the source position determination could be due to systematic antenna-based and baseline-based phase-difference errors. To investigate this source of error we introduced a random phase error for each antenna (constant for each antenna throughout the experiment) of magnitude between 0 and 5 degrees (or equivalently between 0 and 10 degrees for baseline-based errors), using a random number generator. For a given magnitude of phase error, the baseline-based errors induce a smaller astrometric error than do the antenna-based ones. Indeed, the worst case is that one in which the largest antenna-based errors are associated with the longest and most sensitive baseline. We analyzed the magnitude of this type of error in our experiment by splitting the data into two sets by baseline. In one we kept the long and sensitive baselines and in the other we did not. The difference between those solutions, about $13 \mu$ as, indicates that in our experiment the antenna-based systematic phase-difference errors are not much larger than 2 degrees.

Figure 16 contains the summary of all our astrometric results. We have also conducted comparable numerical experiments with the 1981 data and obtained similar results (data quantity and quality, and source structure are similar for both experiments). Hence, we conclude that systematic errors at epochs March 1981 and May 1983 are likely similar.

\section{CONCLUSIONS}

The analysis of systematic errors shows that they are about three times larger than the statistical standard errors. This analysis allows us to conclude that indeed we have detected a significant shift of $57 \pm 17 \mu$ as in the apparent location of the core of $1038+528 \mathrm{~A}$ during a time interval of about two years. The location of the core in May 1983 appears toward the NW (P.A. $-20^{\circ}$ ) of its location in March 1981, thus corresponding to a relative proper motion at $\lambda=3.6 \mathrm{~cm}$ of the core of $1038+528 \mathrm{~A}$ with respect to $1038+528 \mathrm{~B}$ of $26 \pm 8 \mu \mathrm{as} / \mathrm{yr}$. A model for the core of $1038+528 \mathrm{~A}$ and the possible significance of the detected proper motion were presented by Marcaide, Elósegui, and Shapiro (1990).

\section{REFERENCES}

Bartel, N., Herring, T. A., Ratner, M. I., Shapiro, I. I., and Corey, B. E. 1986, Nature, 319, 733.

Marcaide, J. M., Shapiro, I. I., Corey, B. E., Cotton, W. D., Gorenstein, M. V., Rogers, A. E. E., Romney, J. D., Schild, R. E., Bàith, L., Bartel, N., Cohen, N. L., Clark, T. A., Preston, R. A., Ratner, M. I., and Whitney, A. R. 1985, Astr. Ap., $142,71$.

Marcaide, J. M., Elósegui, P., and Shapiro, I. I. 1990, in Parsec Scale Radio Jets, ed. J. A. Zensus and T. J. Pearson, Cambridge University Press, p. 43.

Morabito, D. D., Niell, A. E., Preston, R. A., Linfield, R. P., Wehrle, A. E., and Faulkner, J. 1986, Astron. J., 01, 1038. 
David Schaffer: The change in position is not necessarily a true motion of the quasar core. It could be caused by structure or opacity effects.

P. Elosegui: Indeed, such possibilities were already pointed out by Marcaide, Elosegui, and Shapiro (1990) (see references). Yet, a new experiment will probably tell which of the alternatives is the correct one.

D. H. Roberts: Can you briefly explain why random calibration errors produce a systematic offset?

P. Elosegui: The peak of brightness of the May 1983 map is defined by two $\delta$-components. The random calibration errors smear energy from the largest $\delta$-component to the other one, thus displacing systematically the position of the new peak of brightness towards a given direction. For March 1981, the peak of brightness of the map is defined by a single $\delta$-component and hence the systematic shift does not take place.

A. Jongeneehen: What is the effect of the uncertainty of the scaling and rotation of the coordinate system between the two epochs on the uncertainty of the differential position?

P. Elosegui: We estimate it as less that 1 microarcsecond 